\title{
Fast and Scalable Low-Order Implicit Unstructured Finite-Element Solver for Earth's Crust Deformation Problem
}

\author{
Kohei Fujita \\ Earthquake Research Institute \& \\ Department of Civil Engineering, The \\ University of Tokyo \\ Advanced Institute for Computational \\ Science, RIKEN \\ fujita@eri.u-tokyo.ac.jp \\ Hikaru Inoue \\ Frontier Computing Center, Fujitsu \\ Limited \\ inoue-hikaru@jp.fujitsu.com
}

\author{
Tsuyoshi Ichimura \\ Earthquake Research Institute \& \\ Department of Civil Engineering, The \\ University of Tokyo \\ Advanced Institute for Computational \\ Science, RIKEN \\ ichimura@eri.u-tokyo.ac.jp \\ Muneo Hori \\ Earthquake Research Institute \& \\ Department of Civil Engineering, The \\ University of Tokyo \\ Advanced Institute for Computational \\ Science, RIKEN \\ hori@eri.u-tokyo.ac.jp
}

\author{
Kentaro Koyama \\ East Japan Business Group, Fujitsu \\ Limited \\ kkoyama@jp.fujitsu.com
}

\begin{abstract}
A low-order implicit unstructured finite-element solver for Earth's crust deformation problem is proposed. The solver scales up to 294,912 CPU cores of K computer with $94.8 \%$ size-up efficiency, enabling solving a 504 billion degrees-of-freedom crust deformation problem with 126 billion second-order tetrahedral elements in $146.5 \mathrm{~s}$ at 0.970 PFLOPS (20.6\% of peak) on 294,912 CPU cores. This is 11.1 times faster than a highly-tuned preconditioned conjugate gradient solver and 2.18 times faster than the state-of-the-art SC14 Gordon Bell Prize Finalist solver. With the proposed solver, we solved a practical 381 billion degrees-of-freedom Eastern Japan crust deformation problem $(576 \mathrm{~km}$ x $800 \mathrm{~km}$ x $400 \mathrm{~km}$ area; resolution of $62.5 \mathrm{~m}$ ) in $252.2 \mathrm{~s}$ using 294,912 CPU cores. Such high-resolution analysis assures the convergence of stress and strain required to evaluate nonlinear material constitutive models at plate boundaries and is expected to become a key tool for physics-based earthquake forecasting.
\end{abstract}

\section{CCS CONCEPTS}

- Computing methodologies $\rightarrow$ Massively parallel algorithms; - Applied computing $\rightarrow$ Earth and atmospheric sciences;

\section{KEYWORDS}

Crust deformation, finite-element method, unstructured mesh, implicit solver

Permission to make digital or hard copies of all or part of this work for personal or classroom use is granted without fee provided that copies are not made or distributed for profit or commercial advantage and that copies bear this notice and the full citation on the first page. Copyrights for components of this work owned by others than ACM must be honored. Abstracting with credit is permitted. To copy otherwise, or republish to post on servers or to redistribute to lists, requires prior specific permission and/or a fee. Request permissions from permissions@acm.org.

PASC '17, June 26-28, 2017, Lugano, Switzerland

(C) 2017 Association for Computing Machinery.

ACM ISBN 978-1-4503-5062-4/17/06 ..\$15.00

https://doi.org/10.1145/3093172.3093236
ACM Reference format:

Kohei Fujita, Tsuyoshi Ichimura, Kentaro Koyama, Hikaru Inoue, Muneo Hori, and Lalith Maddegedara. 2017. Fast and Scalable Low-Order Implicit Unstructured Finite-Element Solver for Earth's Crust Deformation Problem. In Proceedings of PASC '17, Lugano, Switzerland, June 26-28, 2017, 10 pages. https://doi.org/10.1145/3093172.3093236

\section{EARTH'S CRUST DEFORMATION PROBLEM}

\subsection{Overview and importance of crust deformation problem}

Earthquakes have caused catastrophic damage throughout history. The process involves faulting, i.e., the release of stress accumulated by plate movement, wave propagation through the Earth's crust, wave amplification at soft soil layers, and seismic structure response (see Fig. 1). These processes are solid continuum mechanics problems of large domains; thus, high-performance computing has played an important role in the analyses of earthquake problems [1-11]. Such studies have enabled high-resolution analyses of earthquake dynamics problems to better understand earthquake damage processes. These methods are now being used by governmental sectors to determine appropriate countermeasures against earthquake disasters.

The next step in earthquake disaster mitigation is forecasting the position, magnitude and time of earthquakes. One approach to this challenging problem is physics-based earthquake forecasting, which is based on assimilation of observation data and high-fidelity analysis $[12,13]$. In such forecasting methods, plate boundary state (cohesive state) change is estimated using GPS crust deformation observation data, and the probability of earthquakes is estimated by comparing the plate boundary state to past earthquake occurrence patterns. The key tool for physics-based forecasting is Earth's crust deformation analysis, which computes the crust deformation for a given fault slip. Analysis results are used with observed crust deformation at surface for inverse analysis to estimate the change in the plate boundary state. Inversion analysis requires accurate 




Figure 1: An earthquake disaster consists of earthquake faulting, wave propagation, soil amplification, and seismic structural response. Physics-based earthquake forecasting, which estimates the cohesive state between plates, is expected to become possible by assimilating GPS crust deformation data with multiple-case crust deformation analysis of $10^{6-7} \times 10^{6-7} \times 10^{4-5} \mathrm{~m}$ at $10^{0-1} \mathrm{~m}$ resolution at the plate boundary.

modeling of the three-dimensional topography geometry and crust layer interfaces, and accurate computation of stress and strain is required to update nonlinear material constitutive models at plate boundaries. As crust deformation analyses is also a large-scale solid continuum mechanics problem, high-performance computing is expected to play an important role in enabling analysis with sufficient accuracy for physics-based earthquake forecasting.

\subsection{Analysis methods for crust deformation problem}

The major cost involved in the crust deformation problem is solving a large linear system of equations governing the force equilibrium in the crust. The time-to-solution of the implicit solver must be kept short to conduct multiple simulations during the data assimilation process; however, an all-purpose solver with short time-to-solution for a wide variety of problems has not yet been developed due to the difficulty in obtaining high peak performance and good load balance while simultaneously achieving good convergency of the iterative solver. One solution is using structured grids, which are straightforward to obtain high performance and good load balance. Although such structured grids are suitable for problems with simple geometry, special consideration is required to obtain accurate stress and strain at complex geometry parts when applied to problems with complex geometry. Using unstructured grids that explicitly discretize complex three-dimensional geometry enables improvement in accuracy, but often leads to poor load balance and lower peak performance. Thus, suitable compute methods are expected to change according to the target problem and the target compute architecture.

To compare the performance of structured and unstructured grids for the crust deformation problem, we solve a crust deformation problem of Eastern Japan using both methods. A $576 \mathrm{~km} \mathrm{x}$ $800 \mathrm{~km} \times 400 \mathrm{~km}$ domain is modeled with eight crust layers with the material properties shown in Table 1, and response for a given fault slip is computed. Figure 2a shows the generated unstructured
Table 1: Material properties of Eastern Japan crust model. Data from J-SHIS [24]. $V_{p}, V_{s}$, and $\rho$ indicate primary wave velocity, secondary wave velocity, and density, respectively.

\begin{tabular}{lrrr}
\hline Layer \# & $V_{p}(\mathrm{~m} / \mathrm{s})$ & $V_{s}(\mathrm{~m} / \mathrm{s})$ & $\rho\left(\mathrm{kg} / \mathrm{m}^{3}\right)$ \\
\hline 1 & 1,600 & 400 & 1,850 \\
2 & 2,000 & 650 & 1,950 \\
3 & 2,600 & 1,100 & 2,150 \\
4 & 3,600 & 1,800 & 2,300 \\
5 & 4,500 & 2,400 & 2,450 \\
6 & 4,800 & 2,800 & 2,500 \\
7 & 5,500 & 3,150 & 2,600 \\
8 & 5,800 & 3,350 & 2,700 \\
\hline
\end{tabular}

finite-element model reflecting the three-dimensional geometry of the crust. Here, the surface is flattened for the structured grid model. Figure $2 \mathrm{~b}$ shows the response of a $125-\mathrm{m}$ structured grid model with 388 billion degrees-of-freedom. This is computed using 294,912 CPU cores of the K computer [14] with measured memory usage of $0.34 \mathrm{~PB}$. The supercomputer with the largest memory (November 2016 Top 500 list [15]) is Sequoia with 1.6 PB memory [16]; thus the scale of this structured grid analysis could be considered a large-scale problem from the perspective of memory usage. Nevertheless, we can see jagged patterns in stress at the crust layer boundaries; therefore, the resolution is insufficient for convergence of stress.

Next, we compute the same problem using unstructured grids with an element size of $125 \mathrm{~m}$ (381 billion degrees-of-freedom; computed using 294,912 CPU cores), element size of $250 \mathrm{~m}$ (degreesof-freedom becomes approximately $1 / 2^{3}=1 / 8$; computed using 36,864 CPU cores), and element size of $500 \mathrm{~m}$ (degrees-of-freedom becomes approximately $1 / 4^{3}=1 / 64$ ). The resolutions of the analyses are $62.5 \mathrm{~m}, 125 \mathrm{~m}$, and $250 \mathrm{~m}$, respectively, because second-order tetrahedral elements are used. As can be seen in Fig. 2b, the stress distribution is smooth for all models, and stress converged with the 250-m element model. The use of second-order tetrahedral elements was sufficient for convergence of stress for this problem. Thus, the low-order unstructured analysis computed using 36,864 CPU cores demonstrates higher accuracy than the structured analysis using 294,912 CPU cores.

From these results, we see that significantly smaller elements are required to obtain equivalent accuracy when using structured grids for this problem. Although there are effective methods to improve the accuracy of structured grid analysis, explicit geometry modeling with unstructured grids is more straightforward. Since the number of elements is proportional to the cubic of the element size for three-dimensional problems, the use of unstructured grids may lead to reduced cost even when considering relatively inefficient data access and computation. As the total memory footprint does not change much regardless of structured/unstructured grids when matrix-free matrix-vector multiplications are used in the solver, the high accuracy per memory footprint capability of unstructured grids is expected to become more important on future systems with relatively low memory capacity. Thus, we target a low-order unstructured finite-element method for the Earth's crust deformation problem in this study. 


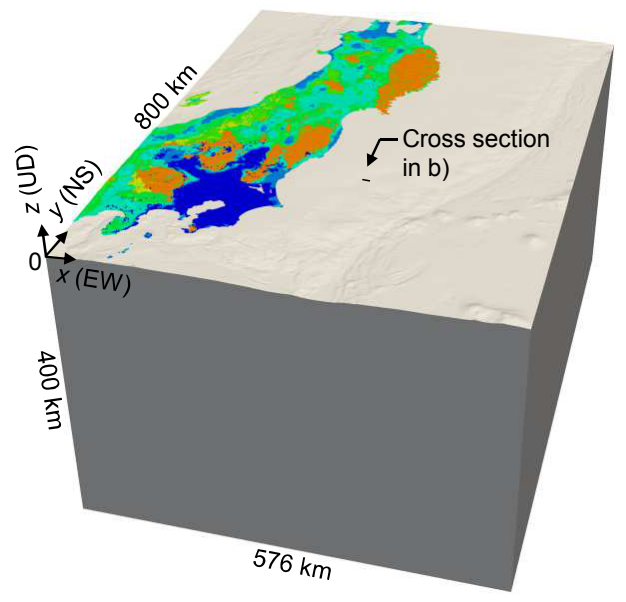

a) Overview of unstructured finite-element model

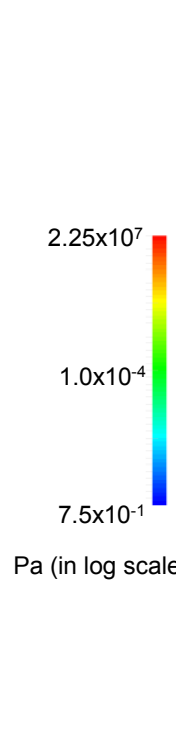

b) Stress response at cross section $y=300 \mathrm{~km}$

Figure 2: Crust deformation analyses for Eastern Japan using structured and unstructured finite-element simulations. a) View of generated unstructured finite-element model. Three-dimensional crust layer geometry and topography is included in the unstructured finite-element model, while the surface is flattened for the structured finite-element model. b) Difference in stress response for structured and unstructured analysis at $y=300 \mathrm{~km}$ plane. All models are computed with the developed second-order tetrahedral finite-element solver (each voxel in structured grid is discretized with six tetrahedral elements).

\subsection{State-of-the-art unstructured analysis and contributions}

Relative to methods that are scalable to full high-performance computer systems with millions of cores, current state-of-the-art loworder unstructured finite-element analysis methods include the Gordon Bell Prize finalists GAMERA and GOJIRA from SC14 and SC15, respectively $[8,9]$. GAMERA uses a multi-grid preconditioner with low cost and high improvement in convergency, together with fast matrix-free matrix-vector multiplication, which enables a low memory footprint and good load balance. This leads to a solver that is scalable to the full $\mathrm{K}$ computer system with short time-tosolution. GOJIRA enhances GAMERA using a hybrid mesh with structured and unstructured elements. In GOJIRA, uniform parts of the target problem are discretized using structured elements, while complex parts are discretized using unstructured elements. This leads to further reduced computation and data access costs for structured parts. An obvious drawback of GOJIRA is that it cannot be applied to a general unstructured tetrahedral mesh; thus, a specialized mesh generator is required. Another state-of-the-art solver is the SC15 Gordon Bell Prize paper [17], which uses a specialized mesh called a forest-of-octree to solve global mantle convection problems. Here, geometry confirming hexahedral mesh is further refined using octrees; thus, it also requires a special mesh rather than a simple tetrahedral mesh. Therefore, we seek to improve a general solver based on GAMERA.
GAMERA was developed for dynamic earthquake wave propagation problems; thus, there is room for improvement for static crust deformation problems. Although sub-optimal for static problems, GAMERA has been applied to a crust deformation problem with a $2,944 \mathrm{~km} \times 2,752 \mathrm{~km} \times 850 \mathrm{~km}$ domain at 500-m resolution using 8,192 compute nodes of the $\mathrm{K}$ computer [18]. The problem size was 10 billion degrees-of-freedom, which is $10^{3-4}$ times larger than conventional crust deformation analyses, but is still 38 times smaller than the $62.5-\mathrm{m}$ resolution unstructured analysis discussed in the previous subsection. Thus, a new solver is required for efficient analysis within limited compute time and memory capacity.

From this perspective, we develop a fast solver suitable for the target static problem with a general unstructured second-order tetrahedral finite-element mesh that is scalable to large high-performance computer systems. With such a solver, we can perform many-case crust deformation analysis that ensures stress/strain convergence using a mesh generated by a wide variety of mesh generators. This is expected to lead to practical inverse analyses of plate boundary state problems on current high-performance computer systems.

The remainder of the paper is organized as follows. Section 2 describes the target problem and the proposed solver algorithm. Section 3 shows a performance comparison of the proposed solver with a standard preconditioned conjugate gradient solver and GAMERA. Section 4 summarizes the paper. 


\section{METHODOLOGY}

\subsection{Target equation}

By approximating crust as an isotropic linear elastic continuum and assuming infinitesimal displacement, the target co-seismic crust deformation problem becomes

$$
\left(c_{i j k l} u_{k, l}\right)_{, i}=f_{j},
$$

where $c$ is the stiffness tensor, and $u$ and $f$ are displacement and outer force vectors, respectively. Subscripts indicate components in a three-dimensional Cartesian coordinate system, and ${ }_{, i}$ is partial differentiation $\frac{\partial}{\partial x_{i}}$. By discretizing Eq. 1 with second-order tetrahedral elements, we obtain;

$$
\mathrm{Ku}=\mathbf{f},
$$

where

$$
\mathbf{K}=\sum_{e} \mathbf{Q}_{e}^{T} \mathbf{K}_{e} \mathbf{Q}_{e}
$$

Here, $\mathbf{K}$ is a symmetric positive definite global stiffness matrix, and $\mathbf{u}, \mathbf{f}, \mathbf{Q}_{e}$, and $\mathrm{K}_{e}$ are an unknown displacement vector, a known force vector, a mapping matrix between an global matrix and an element matrix, and an element stiffness matrix, respectively. Our target is to solve Eq. 2 in the shortest time using high-performance computers.

\subsection{Solver algorithm}

The key components to a fast unstructured low-order finite-element solver on recent large-scale parallel computers are as follows:

(1) a preconditioner with low cost and high convergence improvement,

(2) fast matrix-vector multiplication,

(3) both of the above with a low memory footprint and good scalability.

1) is required to reduce the total number of iterations of the solver, and 2) is required to reduce the cost per iteration. 3) is required to obtain sufficient performance on modern high-performance computers with relatively low memory consisting of many parallel components. Obtaining good scalability requires good load balance as well as small and infrequent communication, which sometimes creates a trade-off between 1) and 2). Thus, overall consideration of the target problem, the target compute system, and the available numerical algorithms are required when designing a solver.

First, we consider a suitable preconditioner for the target problem. Since a preconditioner essentially approximates an inverse of governing Eq. 1, considering the Green's function of the target problem can lead to insight relative to a good preconditioner While Green's function of a dynamic problem has influence area proportional to time step size, Green's function of a static problem becomes infinite. Thus, for a static problem, we must collect information from distant points to obtain a solution at a given point. Since iterative solvers widely used to solve large-scale problems tend to resolve local errors but fail to resolve long range errors with a small number of iterations, a preconditioner that can effectively reduce long range error would be suitable for this problem. An effective solution for this problem is using multi-grids, which use coarse grids to solve long range errors and fine grids to solve local errors. Here, target problem Eq. 2 is converted to solving the following:

$$
\left(\mathbf{P}^{T} \mathbf{K P}\right) \mathbf{u}_{c}=\left(\mathbf{P}^{T} \mathbf{f}\right),
$$

and its solution $\mathbf{u}_{c}$ is used to approximate $\mathbf{u}$ as follows:

$$
\mathbf{u} \approx \mathbf{P u}_{c} .
$$

Here, $\mathbf{P}$ is a matrix that maps between the original grid $\mathbf{u}$ and the generated coarse grid $\mathbf{u}_{c}$. Equations 4 and 5 are used recursively to coarsen problem to a given size. Since the total problem size is decreased on the coarse grid, fewer iterations are required to reduce long range errors.

With structured grids, the use of geometric multi-grids (i.e., equally spaced coarsening for $\mathbf{P}$ ) often leads to an approximate solution $\mathrm{Pu}_{c}$ that is very close to the exact solution $\mathbf{u}$. Thus, by using a multi-grid, long range errors can be reduced effectively on coarse grids with a small number of iterations with small compute and communication size per iteration, while local errors can be solved on the original mesh with fewer iterations, thereby leading to significant speedup of the entire solver. In addition, the matrixvector multiplication used to solve Eq. 4 can often be computed easily on the fly. For example, $\mathbf{P}^{T} \mathbf{K P}$ of a structured grid is equivalent to $\mathrm{K}$ of a structured grid with twice the grid spacing. Thus, matrix-free multiplication can be used rather than precomputing, storing and reading the global matrix from memory each time in an iterative solver. Since it is straightforward to obtain good load balance by allocating an equal number of elements per core, matrixfree multiplication achieves good scalability. This leads to a smaller memory footprint, less memory transfers, and good scalability for the coarse grid preconditioner.

However, for unstructured grids, geometric multi-grids cannot be used (i.e., there is no concept of equally spaced coarsening); thus, $\mathbf{P}$ must be chosen in other ways (i.e., an algebraic multi-grid). While this approach can be used to approximate $\mathbf{u}$ for general unstructured problems, its approximation accuracy is dependent on the choice of $\mathbf{P}$ and is often not as accurate as that of a geometric multi-grid. In addition, matrix-free matrix-vector multiplication of $\left(\mathbf{P}^{T} \mathbf{K P}\right) \mathbf{x}_{c}$ is often very costly; thus, $\mathbf{P}^{T} \mathbf{K P}$ is precomputed and stored in memory. This is a drawback when considering recent computes with relatively low memory access bandwidth and smaller memory capacity.

The key to an effective preconditioner for the crust deformation problem is to combine the efficiency of a geometric multi-grid and the flexibility of algebraic multi-grids to approximate long range errors with low computational cost, less communication and a low memory footprint. Combining a matrix-free matrix-vector multiplication method is expected to achieve good scalability and fast time-to-solution with a low memory footprint. From this perspective, we developed the solver shown in Algorithms 1 and 2. The details of the solver are as follows.

Adaptive conjugate gradient method. Rather than multiplying a fixed matrix that approximates the inverse of matrix $\mathrm{K}$ for a preconditioner (i.e., $\mathbf{z} \Leftarrow \mathrm{Cr}$, where $\mathrm{C} \approx \mathrm{K}^{-1}$ ), another solver is used to solve the preconditioning matrix equation $(\mathrm{Kz}=\mathbf{r})$ using an adaptive conjugate gradient method [19]. We refer to the iterations of the original conjugate gradient loop as the outer loop (Algorithm 1, lines 20-30), and refer to iterations for solving the preconditioning 


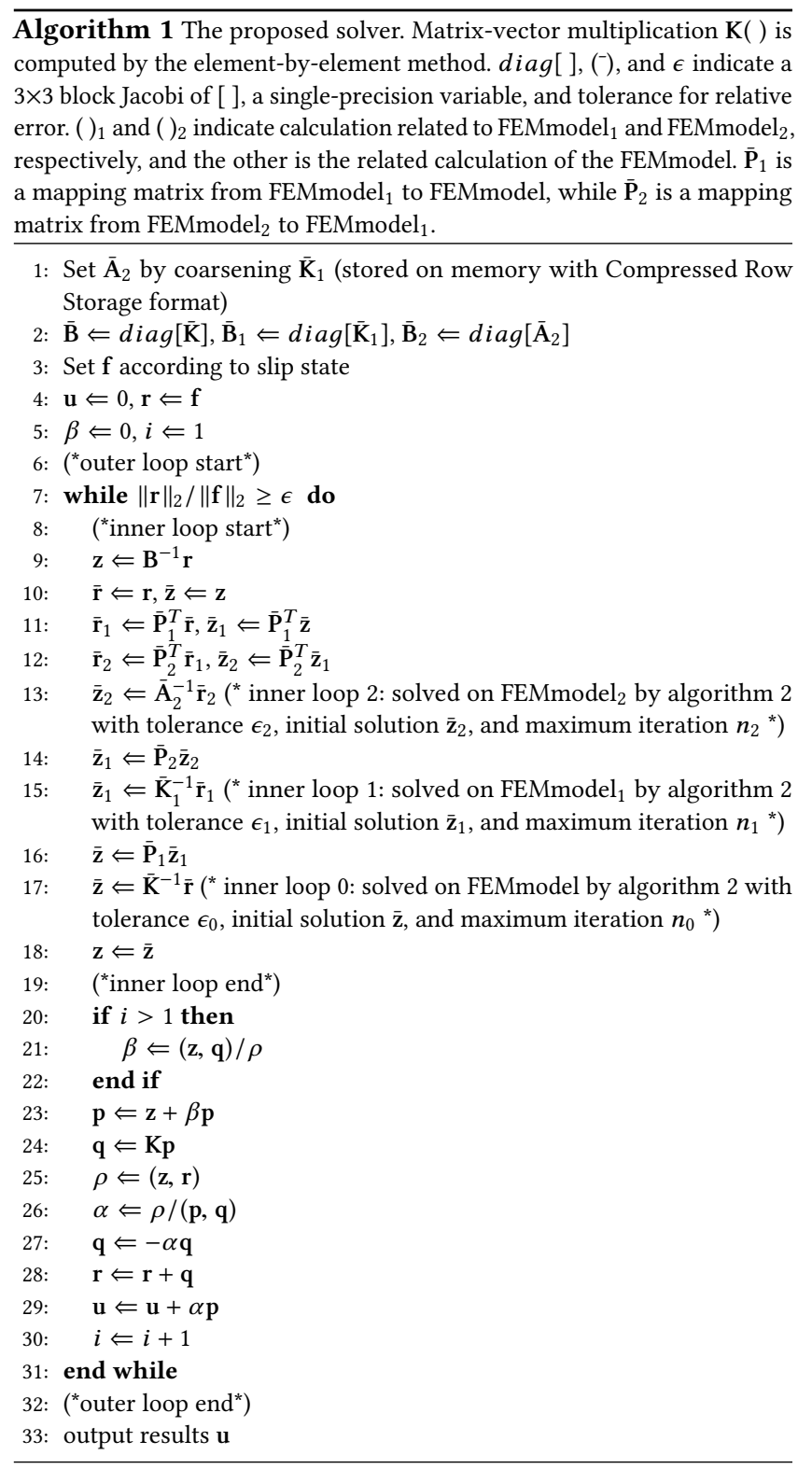

matrix equation as the inner loop (Algorithm 1, lines 8-19). Since the preconditioning matrix equation only needs to be solved approximately, fast methods with limited accuracy can be used for the inner loop. Thus, although the inner loop incurs additional cost, a good inner loop solver can reduce the total number of iterations of the more costly outer loop, which speeds up the solver overall.

Integrated geometric/algebraic multi-grid preconditioner. To utilize the efficiency of a geometric multi-grid and the flexibility of algebraic multi-grids, we combine these methods in the inner loop solver. The idea here is that a second-order tetrahedral element mesh still maps to a linear tetrahedral mesh geometrically even if the mesh itself is unstructured. Thus, we apply geometric coarsening from the targeted second-order tetrahedral mesh to a linear tetrahedral mesh. Then, we apply an algebraic multi-grid to the
Algorithm 2 Conjugate gradient solver with a preconditioner. This is used to roughly solve $\overline{\mathbf{z}}_{2}=\overline{\mathbf{A}}_{2}^{-1} \overline{\mathbf{r}}_{2}, \overline{\mathbf{z}}_{1}=\overline{\mathbf{K}}_{1}^{-1} \overline{\mathbf{r}}_{1}$, and $\overline{\mathbf{z}}=\overline{\mathbf{K}}^{-1} \overline{\mathbf{r}}$ in Algorithm 1. $\mathrm{B}$ and $\epsilon$ are a block Jacobi matrix and the tolerance for the relative error, respectively. $n$ indicates the maximum loop iterations. Matrix-vector multiplications $\mathbf{K}()$ and $\mathrm{K}_{1}($ ) are computed using the element-by-element method for FEMmodel and FEMmodel ${ }_{1}$, while matrix-vector multiplication $\mathbf{A}_{2}(\mathrm{)})$ is computed by reading the global matrix stored in compressed row storage format for $\mathrm{FEMmodel}_{2}$. All calculations are performed using single-precision variables.



linear tetrahedral mesh. The advantages of this approach compared to using pure algebraic multi-grids are threefold. The first is good approximation accuracy of geometric coarsening which leads to less iterations of the fine grid solver. The second is applicability of matrix-free matrix-vector multiplication in the linear tetrahedral solver (i.e., $\mathbf{P}^{T} \mathbf{K P}$ of the second-order tetrahedral mesh is equivalent to $\mathrm{K}$ of the linear tetrahedral mesh), which leads to reduction in memory footprint and memory access costs. The third is reduction of the large memory footprint required for algebraic multi-grids. After geometric coarsening, the grid is significantly smaller than the original grid. For example, the degrees-of-freedom for a typical second-order tetrahedral mesh are reduced to $1 / 8$ by geometric coarsening and are further reduced to 1/64 after additional algebraic coarsening. This enables practical use of multi-grids for solving large-scale problems on machines with limited memory capacity. The communication size is also decreased to $1 / 4$ by geometric coarsening and $1 / 16$ after algebraic coarsening.

A typical way to use geometric multi-grids is using a V-cycle or $\mathrm{W}$-cycle solver. However, the limited approximation accuracy of algebraic multi-grids becomes a bottleneck when frequent mapping occurs between grids. Thus, we use a simple but robust approach for the inner loop solver. Here, we first solve the preconditioning matrix equation roughly on the coarsest grid $\left(\mathrm{FEMmodel}_{2}\right.$; Algorithm 1, line 13) using an iterative solver. Then, we use the obtained solution as an initial solution for the linear tetrahedral model (FEMmodel ${ }_{1}$; Algorithm 1, line 15). Finally, this solution is used as an initial solution to solve the preconditioning matrix equation on the original mesh (FEMmodel). With this approach, we can reduce the frequency of mapping between grids and reduce the overall 
cost of the inner loop solver. In other words, long range errors are solved first on the coarsest grid, and the solution is refined as it proceeds to finer grids. Hereafter, we refer to the solver for the coarsest loop as inner loop level 2, the solver for the linear mesh as inner loop level 1, and the solver for the second-order mesh as inner loop level 0. A standard 3 x 3 block Jacobi preconditioned conjugate gradient solver (Algorithm 2) is used to solve all inner loops.

Although implementation of inner loop levels 0 and 1 are straightforward, implementation of the algebraic multi-grid (inner loop level 2) in a distributed memory parallel environment must be considered carefully. Here, we use $\mathrm{C}$ nodes (nodes that remain in FEMmodel $_{2}$ ) and $\mathrm{F}$ nodes (nodes eliminated in $\mathrm{FEMmodel}_{2}$ ) such that adjacent MPI partitions have consistent $\mathrm{C}$ and $\mathrm{F}$ nodes across the MPI boundaries. Here, we first color the MPI boundary nodes synchronously among MPI processes, and then we color the internal nodes per process. This enables coarsening of internal and MPI boundary nodes, which leads to smaller matrix and vector sizes and less MPI communication in the level 2 solver.

Element-by-element method. The element-by-element (EBE) method [20] is a matrix-free method that computes matrix-vector multiplication $\mathrm{y} \Leftarrow \mathrm{Kx}$ element wise as follows:

$$
\mathrm{y} \Leftarrow \sum_{e}\left(\mathbf{Q}_{e}^{T}\left(\mathrm{~K}_{e}\left(\mathbf{Q}_{e} \mathbf{x}\right)\right)\right) .
$$

$\mathrm{K}_{e}$ can be generated from a small amount of information (i.e., node coordinates and material property); thus, the EBE method can be considered a type of matrix-vector multiplication that converts part of the memory access cost of standard matrix-vector multiplication (i.e., reading a global matrix from memory) to in-cache compute cost. Compared to standard matrix-vector multiplication involving nonuniform memory access that often causes load imbalance among parallel processing elements, the EBE method can obtain good load balance by allocating an equal number of elements per core. Thus, the EBE method is expected to enable shorter time-to-solution when used on modern massively-parallel computers with relatively low B/F capability. In the computation of Eq. 6, we use multi-core coloring and SIMD buffering methods to utilize multi-core and SIMD parallel compute elements with software prefetching to hide memory access latency. This highly-tuned EBE kernel is used for matrix-vector multiplication in the outer loop (Algorithm 1, line 24) and inner loop levels 0 and 1 (Algorithm 2, line 2 and 12). Since the EBE method cannot be used for inner loop level 2, the global matrix is stored and read from memory for this case (Algorithm 2, line 2 and 12).

Mixed precision arithmetic. Although double precision is required for the final solution in crust deformation analysis, the preconditioner can be computed in single precision because it only needs to be computed roughly. Thus, we use single-precision arithmetic in the inner loops, and the outer loop is computed in double precision. By setting a suitable threshold for the inner loops, we can shift nearly all computation to the inner loops. This halves the memory footprint, memory transfer size, and communication size, and doubles the apparent cache size. Since recent compute architectures have higher compute capability for single-precision floating point arithmetic, we can also expect higher peak performance.
In summary, we have developed a method that combines the strengths of geometric and algebraic multi-grids for low total compute cost for an unstructured finite-element crust deformation problem. Furthermore, solver components are computed using fast, scalable, and memory-efficient kernels, which leads to high performance on modern multi-core and SIMD-based massively-parallel computer systems. In the following section, we evaluate the performance of the proposed solver on the K computer and an Intel Xeon cluster system.

\section{PERFORMANCE MEASUREMENT}

\subsection{Large-scale problem performance on $\mathrm{K}$ computer}

Targeting large-scale problems, here, we measure performance on the K computer system at RIKEN, Advanced Institute for Computational Science [14]. The K computer is a massively-parallel supercomputer comprising 82,944 compute nodes, each with a single SPARC64 VIIIfx CPU. Each of the eight cores of the SPARC CPU has two sets of fused multiply-add (FMA) SIMD units of length 2. This leads to FMA (two operations) $\mathrm{x}$ SIMD length $2 \times 2$ sets $=8$ operations per clock. Consequently, the peak performance of each CPU is 8 operations $\times 8$ cores $\times 2 \mathrm{GHz}=128$ GFLOPS. The SIMD length is 2 for both single- and double-precision arithmetic; thus the peak performance of single-precision arithmetic is also 128 GFLOPS. Each core has two 32-KB two-way L1 caches, one for data and one for instructions, and the the cores share a 6-MB 12-way L2 cache (shared with data/instruction). Each node has 16 GB of DDR3 SDRAM memory with a peak memory bandwidth of 64 $\mathrm{GB} / \mathrm{s}$. Tofu, a six-dimensional interconnection network, is used for communication between nodes [21]. Each node can communicate in four directions simultaneously with $5-\mathrm{GB} / \mathrm{s}$ throughput in each direction. An OpenMPI [22]-based MPI library optimized for the Tofu network is used.

With this system, we compare the performance of the proposed solver to PCGE and GAMERA. PCGE is a preconditioned conjugate gradient solver with a $3 \times 3$ block Jacobi preconditioner using the EBE method, which was considered a standard solver in SC14 and SC15 papers [8, 9]. This is equivalent to skipping lines 10-18 in Algorithm 1. GAMERA is the SC14 Gordon Bell Prize Finalist solver [8] and uses a two-step geometric multi-grid and mixed-precision arithmetic. This is equivalent to skipping lines 12-14 in Algorithm 1. Both PCGE and GAMERA implementations were generated by skipping the appropriate lines in the proposed solver. Thus, all implementations use the highly-tuned EBE kernel for matrix-vector multiplication. We solve all problems with relative error tolerance $\epsilon=1.0 \times 10^{-8}$. The inner loop tolerances and maximum iterations used for the proposed solver and GAMERA are summarized in Table 2. All weak and strong scaling problems in the following were measured using a two-layered horizontally-stratified crust structure with the material properties summarized in Table 3. A periodic fault input and boundary condition setting was used to make the problem suitable for measuring weak scaling. Although a periodical setting, METIS 5.1.0 [23] was used to partition the mesh for distributed memory computation; we can expect load-balancing and communication characteristics similar to an actual problem. 
Table 2: Error tolerance $\epsilon_{i}$ and maximum iteration $n_{i}$ used for the proposed solver and GAMERA for solving measurement models and application problem

\begin{tabular}{lrrrrrr} 
& $\epsilon_{0}$ & $\epsilon_{1}$ & $\epsilon_{2}$ & $n_{0}$ & $n_{1}$ & $n_{2}$ \\
\hline Developed solver & 0.1 & 0.05 & 0.025 & 30 & 300 & 3,000 \\
GAMERA & 0.1 & 0.05 & - & 30 & 3,000 & - \\
\hline
\end{tabular}

Table 3: Material properties of performance measurement models $\left(V_{p}, V_{s}\right.$, and $\rho$ indicate primary wave velocity, secondary wave velocity, and density, respectively)

\begin{tabular}{lrrr}
\hline Layer \# & $V_{p}(\mathrm{~m} / \mathrm{s})$ & $V_{s}(\mathrm{~m} / \mathrm{s})$ & $\rho\left(\mathrm{kg} / \mathrm{m}^{3}\right)$ \\
\hline 1 & 1,600 & 400 & 1,850 \\
2 & 5,800 & 3,350 & 2,700 \\
\hline
\end{tabular}

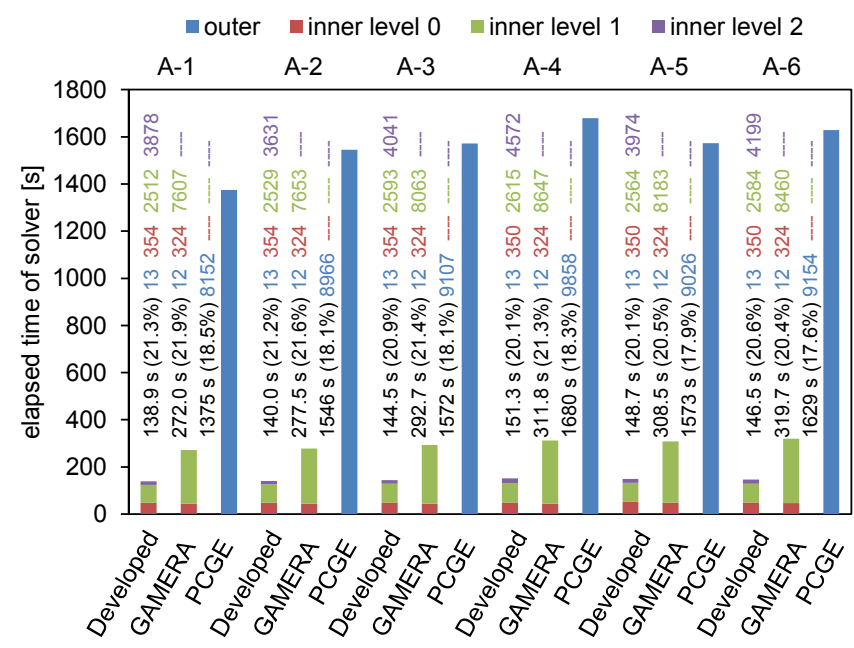

Figure 3: Size-up scalability for model set A measured on the $K$ computer (numbers indicate elapsed time, floating-point arithmetic efficiency to peak, and elapsed iterations for the outer loop, and inner level 0, 1, and 2 loops)

MPI was used for inter-node communication and eight OpenMP threads were used per node for intra-node computation.

3.1.1 Weak scaling up to 0.5 TDOF on 294,912 CPU cores. We first measured weak scaling by fixing the problem size per MPI process to 13.7 million degrees-of-freedom (DOF) using model set A (Table 4). These models are intended to measure the performance of largest-scale problems within available memory. The number of PCGE iterations required for convergence for these models does not change significantly (8,152 to 9,858 iterations); thus, this model set is suitable for measuring size-up scalability. Figure 3 shows the performance of the proposed solver. The number of iterations required for convergence was approximately constant for models A-1 (9,216 CPU cores) to A-6 (294,912 CPU cores), and the size-up efficiency in terms of elapsed time was $94.8 \%$. This high scalability leads to a high compute efficiency (20.6\%; 0.970 PFLOPS) with the largest model measured using 294,912 CPU cores.

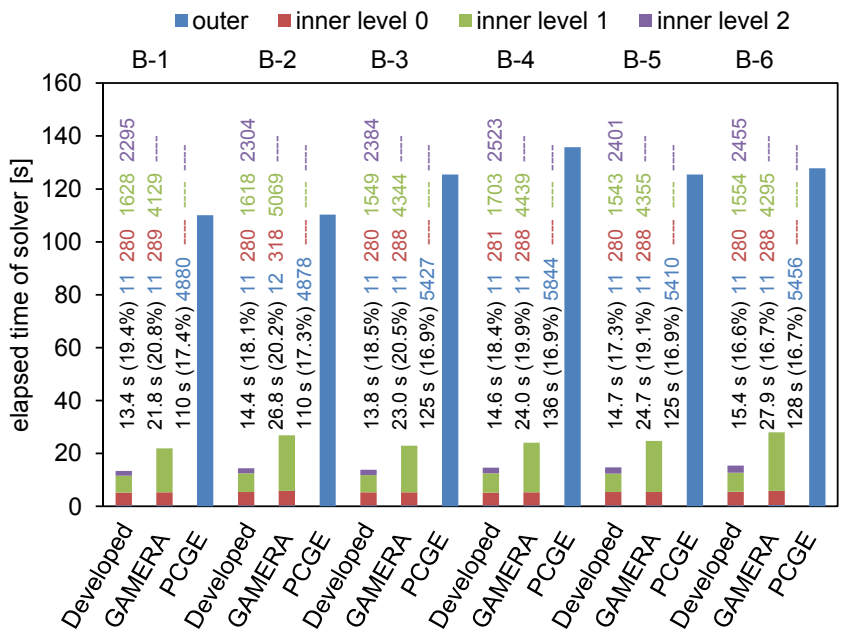

Figure 4: Size-up scalability for model set B measured on the $K$ computer (numbers indicate elapsed time, floating-point arithmetic efficiency to peak, and elapsed iterations for the outer loop, inner level 0,1 , and 2 loops)

Next, we compare the performance of PCGE and GAMERA with model A-6 (i.e., the largest model). Relative to the total number of iterations required for convergence, PCGE required 9,154 iterations, GAMERA required 12 (outer) + 324 (inner level 0$)+8,460$ (inner level 1) $=8,796$ iterations, and the proposed solver required 13 (outer) + $350($ inner level 0$)+2,584($ inner level 1) $+4,199($ inner level 2) $=$ 7,146 iterations. From these results, we see that use of the multi-grid is effective at reducing long range errors in fewer total iterations. With mixed-precision arithmetic and smaller grid size (degrees-offreedom for MPI rank \#0 was reduced from FEMmodel: 13,911,087 $\rightarrow$ FEMmodel $_{1}: 1,772,985 \rightarrow$ FEMmodel $\left._{2}: 270,795\right)$, elapsed time per iteration decreased from $0.18 \mathrm{~s}$ for the outer loop to $0.13 \mathrm{~s}$ (inner level 0), $0.032 \mathrm{~s}$ (inner level 1), and $0.0046 \mathrm{~s}$ (inner level 2). This led to further overall speedup of the solver, i.e., the solver was 11.1 times faster than PCGE and 2.18 times faster than GAMERA.

3.1.2 Weak scaling up to 0.063 TDOF on 294,912 CPU cores. Next we measured weak scaling for a smaller problem (1.7 million degrees-of-freedom per MPI process) using model set $\mathrm{B}$. These models are intended to measure the performance of problems where many simulations must be run for data assimilation. The iterations required for convergence with the PCGE solver did not change significantly (4,880 to 5,844 iterations); thus, this model set is also suitable for measuring size-up scalability. Fig. 4 shows the performance of the proposed solver. We can see that the number of iterations required for convergence was nearly constant among the models. The size-up efficiency was $87.0 \%$ from 9,216 CPU cores (B-1) to 294,912 CPU cores (B-6). Although slightly less than the performance obtained with model A-6, the peak performance with model B-6 was $16.6 \%$ of peak (0.783 PFLOPS), which is still high for an unstructured low-order finite-element solver. The degradation in performance could be due to the smaller problem size, which leads to relatively longer ratios of communication and data transfer latency time relative to the total elapsed time. The speedup obtained 
Table 4: Model configurations (model sets A and B are weak-scaling model sets with different problem sizes per node; model set $\mathrm{C}$ is a strong-scaling model set)

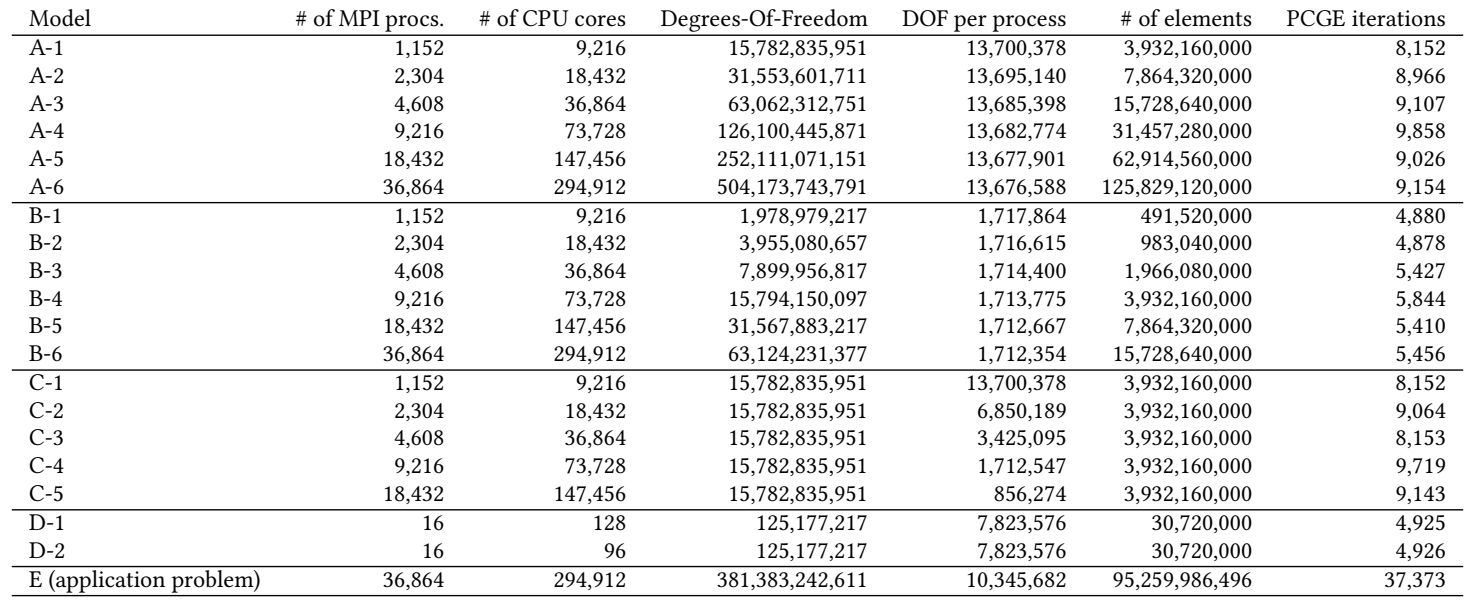

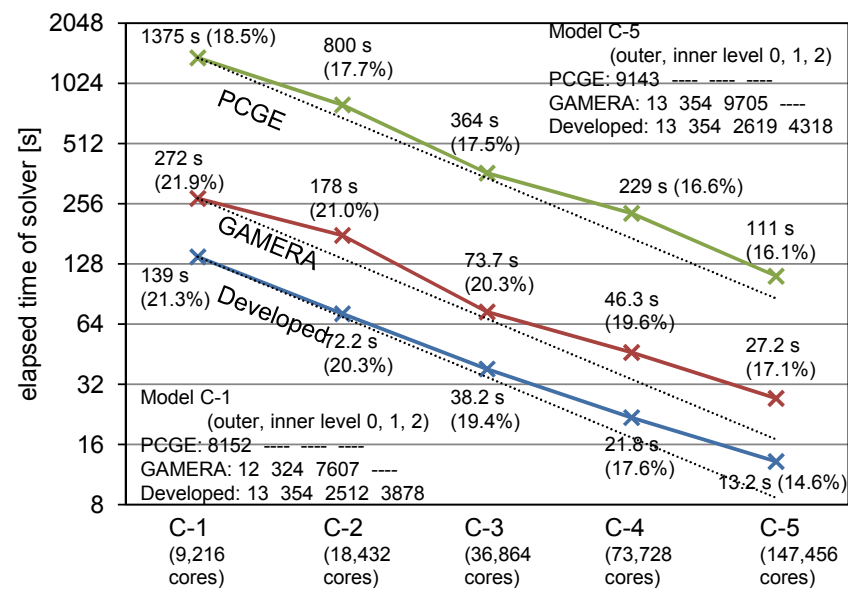

Figure 5: Speed-up scalability for model set $\mathrm{C}$ measured on the $K$ computer (insets indicate elapsed time and floatingpoint arithmetic efficiency to peak; the number of iterations for models $\mathrm{C}-1$ and model $\mathrm{C}-5$ is indicated in the bottom left and top right corners; the dashed lines indicate ideal scaling)

from PCGE and GAMERA was 8.30 and 1.81 times, respectively, for this model.

3.1.3 Strong scaling. Next, we measured strong scaling using the same model as A-1 while varying the number of CPU cores. Figure 5 shows the results. The solver scales well up to 147,456 CPU cores with speedup efficiency of $65.7 \%$, and obtains nearly constant speed-up ratios with PCGE and GAMERA for all problem sizes. Such good scalability is expected to be due to the solver algorithm with good load-balancing characteristics.

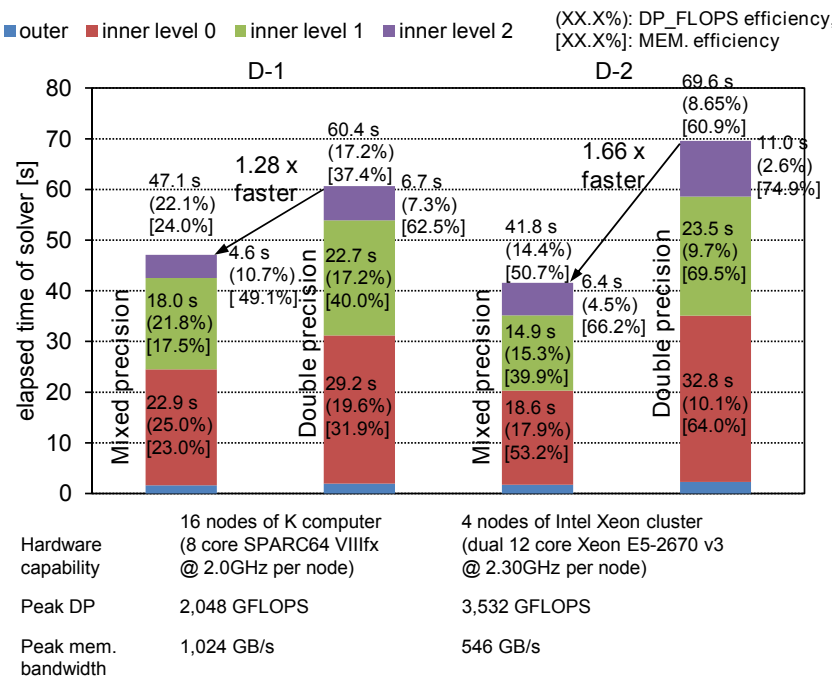

Figure 6: Performance comparison with Intel Xeon cluster and $K$ computer using model set $D$ (plain numbers indicate elapsed time, numbers in brackets indicate performance efficiency to peak DP_FLOPS capability, and numbers in square brackets indicate memory bandwidth utilization; FLOPS and memory bandwidth on the Xeon cluster were approximated by assuming that FLOP count and memory transfer size is equal to that of the $K$ computer)

\subsection{Performance portability on Intel Xeon cluster}

Intel CPU-based clusters are widely used by scientists in the solid earth science field, which are the primary target users of the proposed solver. As an example of such systems, we measured performance on an Intel Xeon Haswell-EP CPU cluster. The main difference from the compute nodes of the $\mathrm{K}$ computer is the longer 
outer $\quad$ inner level $0 \quad$ inner level $1 \quad$ inner level 2

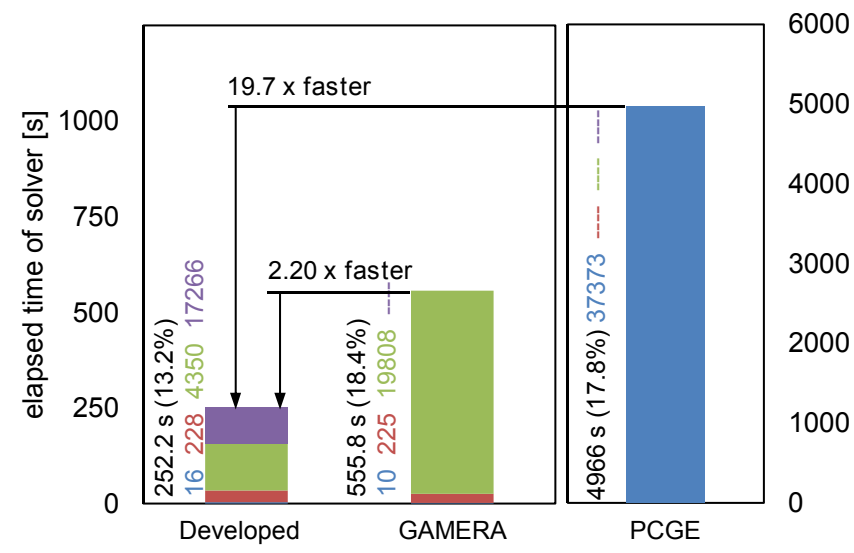

Figure 7: Performance of application problem on the $\mathrm{K}$ computer (insets indicate elapsed time, floating-point arithmetic efficiency to peak, and number of outer loops, and inner level 0,1 , and 2 loops, respectively)

SIMD width (increased from 128 bits to 256 bits) and lower memory bandwidth capability (bytes/DP_FLOPS is reduced from 0.5 of the $\mathrm{K}$ computer to 0.15 ). Here, we solved the same problem with 16 nodes of the K computer with total hardware capability of 2048 DP_TFLOPS, 2048 SP_TFLOPS, and 1024-GB/s memory bandwidth and four nodes of dual Intel Xeon E5-2670 v3 cluster with total hardware capability of 3532 DP_TFLOPS, 7064 SP_TFLOPS, and 546-GB/s memory bandwidth. The same code was used for both systems, with additional SIMD directives for the Xeon cluster to request AVX2 SIMD instructions from the Intel compiler (version 17.0.0) Figure 6 shows the performance results. The compute-intensive solvers using the EBE method (inner loops 0 and 1) were faster on the Xeon cluster, while the memory-access-intensive solver (inner loop 2) was faster on the K computer. Thus, the solver achieves performance increase according to the hardware capability of the target compute system. In total, the solver obtained $14.4 \%$ of DP_peak with the Xeon cluster for the total solver.

To assess the effectiveness of mixed-precision arithmetic, we compared performance with a solver without mixed-precision arithmetic (i.e., all computation was performed in double precision). The speedup obtained using mixed-precision arithmetic was 1.28 times on the K computer and 1.66 times on the Xeon cluster. The mixedprecision arithmetic algorithm effectively reduced memory access cost for both systems, as well as the effective use of wider SIMD lanes with the Xeon cluster. Such effective speedup can also be expected on other machines with higher single-precision compute capabilities.

\subsection{Practical problem performance}

Last, we measured performance with the Eastern Japan problem discussed in Section 1. This unstructured finite-element model includes a three-dimensional crust structure consisting of eight layers, and its resolution is higher than the other measurement models (resolution is $62.5 \mathrm{~m}$ ). Figure 7 shows the performance results for the proposed solver when running on 294,412 CPU cores of the $\mathrm{K}$ computer (model $\mathrm{E}$ in Table 4). Although the floating-point performance efficiency decreased to $13.2 \%$ in this case, the speedup from PCGE increased to 19.7 times due to the increase in the ratio of time used for the memory bandwidth intensive inner level 2 solver, which is more effective with actual high-resolution problems that are more difficult to converge with fine-grid solvers. The speedup from GAMERA was 2.20 times in this case. The time to prepare the preconditioner and output the results took $57.9 \mathrm{~s}$ (proposed solver), $20.7 \mathrm{~s}$ (GAMERA) and $20.3 \mathrm{~s}$ (PCGE), resulting in total times of $310.1 \mathrm{~s}, 576.5 \mathrm{~s}$, and $4986 \mathrm{~s}$, respectively. Thus, the proposed solver obtains speedup relative to both hypothetical performance measurement models and actual crust deformation problems, even when including additional costs required for preparing the level 2 matrix (Algorithm 1, line 1).

\section{CLOSING REMARKS}

We have proposed a fast and scalable low-memory footprint implicit unstructured finite-element solver for crust deformation problems. The proposed solver obtained $94.8 \%$ size-up efficiency with up to 294,912 CPU cores of K computer with performance of 0.97 PFLOPS ( $20.6 \%$ of peak). This is 11.1 faster than a previously proposed highlytuned preconditioned conjugate gradient solver and 2.18 times faster than the previous state-of-the-art solver from SC14. The proposed solver also obtained a high speed-up rate in practical application, i.e., 19.7 times speedup over preconditioned conjugate gradient solver and 2.20 times speedup over the SC14 solver. We combined an adaptive conjugate gradient method, a geometric and algebraic multi-grid method, mixed-precision arithmetic, and fast matrix-free matrix-vector multiplication methods to use the diverse accuracy spaces of numerical methods and several precision spaces of the hardware to effectively solve the wide solution space of the crust deformation problem. The assurance of convergence of stress and strain at plate boundaries is expected to enable more reliable estimations of plate boundary state, and is expected to become a key tool for earthquake forecasting simulations in the future.

\section{ACKNOWLEDGMENTS}

Results were obtained using the K computer at the RIKEN Advanced Institute for Computational Science (Proposal numbers: hp160221, hp160160, and hp160157). We acknowledge the support from Japan Society for the Promotion of Science (15K18110, 26249066, and 25220908) and the FOCUS Establishing Supercomputing Center of Excellence. We used Japanese crust data from J-SHIS of the National Research Institute for Earth Science and Disaster Prevention.

\section{REFERENCES}

[1] Dimitri Komatitsch, Seiji Tsuboi, Chen Ji and Jeroen Tromp, A 14.6 billion degrees of freedom, 5 teraflops, 2.5 terabyte earthquake simulation on the Earth Simulator, in: Proceedings of the ACM / IEEE Supercomputing SC'2003 conference, p. 4-11, doi: 10.1145/1048935.1050155 (2003).

[2] T. Tiankai, Y. Hongfeng, L. Ramirez-Guzman, J. Bielak, O. Ghattas, M. Kwan-Liu, and D. R. O'Hallaron, "From mesh generation to scientific visualization: an endto-end approach to parallel supercomputing," Proceedings of the $2006 \mathrm{ACM} / \mathrm{IEEE}$ conference on Supercomputing (SC '06). ACM, New York, NY, USA, 2006, Article 91. DOI $=10.1145 / 1188455.1188551$

[3] Laura Carrington, Dimitri Komatitsch, Michael Laurenzano, Mustafa Tikir, David Michea, Nicolas Le Goff, Allan Snavely and Jeroen Tromp, High-frequency simulations of global seismic wave propagation using SPECFEM3D_GLOBE, in: 
Proceedings of the ACM / IEEE Supercomputing SC'2008 conference, article \#60, p. 60:1-60:11, doi: 10.1145/1413370.1413432 (2008).

[4] Y. Cui, K. B. Olsen, T. H. Jordan, K. Lee, J. Zhou, P. Small, D. Roten, G. Ely, D. K. Panda, A. Chourasia, J. Levesque, S. M. Day and P. Maechling, "Scalable Earthquake Simulation on Petascale Supercomputersm," Proceedings of the 2010 ACM/IEEE International Conference for High Performance Computing, Network ing, Storage and Analysis (SC '10). IEEE Computer Society, Washington, DC USA, 2010, pp.1-20. DOI=10.1109/SC.2010.45 http://dx.doi.org/10.1109/SC.2010.45

[5] M. Rietmann, P. Messmer, T. Nissen-Meyer, D. Peter, P. Basini, D. Komatitsch O. Schenk, J. Tromp, L. Boschi, and D. Giardini. "Forward and adjoint simulations of seismic wave propagation on emerging large-scale GPU architectures,' Proceedings of the International Conference on High Performance Computing, Networking, Storage and Analysis (SC '12). IEEE Computer Society Press, Los Alamitos, CA, USA, 2012, Article 38, 11 pages.

[6] Y. Cui, E. Poyraz, K. B. Olsen, J. Zhou, K. Withers, S. Callaghan, J. Larkin, C. Guest, D. Choi, A. Chourasia, Z. Shi, S. M. Day, P. J. Maechling and T.H. Jordan. "Physics-based seismic hazard analysis on petascale heterogeneous supercomputers," Proceedings of the International Conference on High Performance Computing, Networking, Storage and Analysis, (SC'13). IEEE Computer Society Press, New York, NY, USA, 2013, Article 70, 12 pages.

[7] A. Heinecke, A. Breuer, S. Rettenberger, M. Bader, A-A. Gabriel, C. Pelties, A Bode, W. Barth, X-K. Liao, K. Vaidyanathan, M. Smelyanskiy, P. Dubey, "Petascale High Order Dynamic Rupture Earthquake Simulations on Heterogeneous Super computers," Proceedings of the International Conference on High Performance Computing, Networking, Storage and Analysis, (SC'14), pp 3-14, 2014.

[8] T. Ichimura, K. Fujita, S. Tanaka, M. Hori, M. Lalith, Y. Shizawa, and H. Kobayashi. "Physics-based urban earthquake simulation enhanced by 10.7 BlnDOF x $30 \mathrm{~K}$ time-step unstructured FE non-linear seismic wave simulation," Proceedings of the International Conference on High Performance Computing, Networking, Storage and Analysis, (SC'14), pp 15-26, 2014.

[9] T. Ichimura, K. Fujita, P.E.B. Quinay, L. Maddegedara, M. Hori, S. Tanaka, Y Shizawa, H. Kobayashi, and K. Minami, "Implicit nonlinear wave simulation with 1.08T DOF and 0.270 T unstructured finite elements to enhance comprehensive earthquake simulation," Proceedings of the International Conference on High Performance Computing, Networking, Storage and Analysis, (SC'15), 2015.

[10] Daniel Roten, Yifeng Cui, Kim B. Olsen, Steven M. Day, Kyle Withers, William H. Savran, Peng Wang, and Dawei Mu. 2016. High-frequency nonlinear earthquake simulations on petascale heterogeneous supercomputers. In Proceedings of the International Conference for High Performance Computing, Networking, Storage and Analysis (SC '16). IEEE Press, Piscataway, NJ, USA, Article 82, 12 pages.

[11] Heinecke A., Breuer A., Bader M., Dubey P. (2016) High Order Seismic Simulations on the Intel Xeon Phi Processor (Knights Landing). In: Kunkel J., Balaji P., Dongarra J. (eds) High Performance Computing. ISC High Performance 2016. Lecture Notes in Computer Science, vol 9697. Springer, Cham.

[12] T. Hori, M. Hyodo, R. Nakata, S. Miyazaki, and Y. Kaneda, "A forecasting procedure for plate boundary earthquakes based on sequential data assimilation," Oceanography 27(2), pp.94-102, 2014.

[13] Takane Hori, Mamoru Hyodo, Shin'ichi Miyazaki, Yoshiyuki Kaneda, Numerical forecasting of the time interval between successive M8 earthquakes along the Nankai Trough, southwest Japan, using ocean bottom cable network data, Marine Geophysical Research, 2014, 35 pp 285-294.

[14] H. Miyazaki, Y. Kusano, N. Shinjou, F. Shoji, M. Yokokawa, and T. Watanabe "Overview of the K computer system," FUJITSU Sci. Tech. J., 48, 3, 302-309, 2012.

[15] Top 500, [Online]. https://www.top500.org/

[16] Sequoia, [Online]. http://computation.llnl.gov/computers/sequoia

[17] J. Rudi, A. Cristiano I. Malossi, T. Isaac, G. Stadler, M. Gurnis, P. W. J. Staar, Y Ineichen, C. Bekas, A. Curioni, O. Ghattas, "An Extreme-Scale Implicit Solver for Complex PDEs: Highly Heterogeneous Flow in Earth's Mantle," Proceedings of the International Conference on High Performance Computing, Networking, Storage and Analysis, (SC'15), 2015.

[18] T. Ichimura, R. Agata, T. Hori, K. Hirahara, C. Hashimoto, M. Hori, and Y. Fukahata, "An elastic/viscoelastic finite element analysis method for crustal deformation using a 3D island-scale high-fidelity model," Geophysical Journal International, doi:10.1093/gji/ggw123 (in advance access, first published online April 4, 2016).

[19] G. H. Golub and Q. Ye Inexact conjugate gradient method with inner-outer iteration. SIAM., Journal on Scientific Computing 21(4), 1305-1320, 1997.

[20] J. M. Winget and T. J. R. Hughes, "Solution algorithms for nonlinear transient heat conduction analysis employing element-by-element iterative strategies, Computer Methods in Applied Mechanics and Engineering, 52, 711-815, 1985.

[21] Y. Ajima, T. Inoue, S. Hiramoto and T. Shimizu. "Tofu: interconnect for the K computer," FUJITSU Sci. Tech. J., Vol.48, No.3, pp.280-285, 2012.

[22] OpenMPI, [Online]. http://www.open-mpi.org/

[23] METIS 5.1.0, [Online] http://glaros.dtc.umn.edu/gkhome/metis/metis/overview

[24] Japan Seismic Hazard Information Station (J-SHIS) [Online]. http://www.j-shis.bosai.go.jp/. 